\title{
BMD-based assessment of local porosity in human femoral cortical bone
}

Citation for published version (APA):

Iori, G., Heyer, F., Kilappa, V., Wyers, C., Varga, P., Schneider, J., Graesel, M., Wendlandt, R., Barkmann, R., van den Bergh, J. P., \& Raum, K. (2018). BMD-based assessment of local porosity in human femoral cortical bone. Bone, 114, 50-61. https://doi.org/10.1016/j.bone.2018.05.028

Document status and date:

Published: 01/09/2018

DOI:

10.1016/j.bone.2018.05.028

Document Version:

Publisher's PDF, also known as Version of record

Document license:

Taverne

Please check the document version of this publication:

- A submitted manuscript is the version of the article upon submission and before peer-review. There can be important differences between the submitted version and the official published version of record.

People interested in the research are advised to contact the author for the final version of the publication, or visit the DOI to the publisher's website.

- The final author version and the galley proof are versions of the publication after peer review.

- The final published version features the final layout of the paper including the volume, issue and page numbers.

Link to publication

\footnotetext{
General rights rights.

- You may freely distribute the URL identifying the publication in the public portal. please follow below link for the End User Agreement:

www.umlib.nl/taverne-license

Take down policy

If you believe that this document breaches copyright please contact us at:

repository@maastrichtuniversity.nl

providing details and we will investigate your claim.
}

Copyright and moral rights for the publications made accessible in the public portal are retained by the authors and/or other copyright owners and it is a condition of accessing publications that users recognise and abide by the legal requirements associated with these

- Users may download and print one copy of any publication from the public portal for the purpose of private study or research.

- You may not further distribute the material or use it for any profit-making activity or commercial gain

If the publication is distributed under the terms of Article $25 \mathrm{fa}$ of the Dutch Copyright Act, indicated by the "Taverne" license above, 
Full Length Article

\title{
BMD-based assessment of local porosity in human femoral cortical bone
}

\author{
Gianluca Iori $^{\mathrm{a}}$, Frans Heyer ${ }^{\mathrm{b}, \mathrm{c}}$, Vantte Kilappa ${ }^{\mathrm{d}}$, Caroline Wyers ${ }^{\mathrm{b}, \mathrm{c}}$, Peter Varga ${ }^{\mathrm{e}}$, \\ Johannes Schneider ${ }^{\mathrm{a}}$, Melanie Gräsel ${ }^{\mathrm{f}}$, Robert Wendlandt ${ }^{\mathrm{g}}$, Reinhard Barkmann ${ }^{\mathrm{f}}$, \\ J.P. van den Bergh ${ }^{\mathrm{b}, \mathrm{c}}$, Kay Raum ${ }^{\mathrm{a}, *}$ \\ a Berlin-Brandenburg Center for Regenerative Therapies, Charité - Universitätsmedizin Berlin, Germany \\ ${ }^{\mathrm{b}}$ Department of Internal Medicine, NUTRIM School of Nutrition and Translational Research in Metabolism, Maastricht University Medical Center, Maastricht, The \\ Netherlands \\ ${ }^{\mathrm{c}}$ Department of Internal Medicine, VieCuri Medical Center, Venlo, The Netherlands \\ ${ }^{\mathrm{d}}$ Mango Solutions, Jyväskylä, Finland \\ e AO Research Institute Davos, Davos, Switzerland \\ ${ }^{\mathrm{f}}$ Sektion Biomedizinische Bildgebung, Klinik für Radiologie, Universitätsklinikum Schleswig-Holstein, Campus Kiel, Germany \\ ${ }^{\mathrm{g}}$ Universitätsklinikum Schleswig-Holstein, Lübeck, Germany
}

\section{A R T I C L E I N F O}

\section{Keywords:}

Cortical bone

Scanning acoustic microscopy

HR-pQCT

Porosity

Image registration

\begin{abstract}
A B S T R A C T
Cortical pores are determinants of the elastic properties and of the ultimate strength of bone tissue. An increase of the overall cortical porosity (Ct.Po) as well as the local coalescence of large pores cause an impairment of the mechanical competence of bone. Therefore, Ct.Po represents a relevant target for identifying patients with high fracture risk. However, given their small size, the in vivo imaging of cortical pores remains challenging. The advent of modern high-resolution peripheral quantitative computed tomography (HR-pQCT) triggered new methods for the clinical assessment of Ct.Po at the peripheral skeleton, either by pore segmentation or by exploiting local bone mineral density (BMD). In this work, we compared BMD-based Ct.Po estimates with highresolution reference values measured by scanning acoustic microscopy. A calibration rule to estimate local Ct.Po from BMD as assessed by HR-pQCT was derived experimentally. Within areas of interest smaller than $0.5 \mathrm{~mm}^{2}$, our model was able to estimate the local Ct.Po with an error of 3.4\%. The incorporation of the BMD inhomogeneity and of one parameter from the BMD distribution of the entire scan volume led to a relative reduction of the estimate error of $30 \%$, if compared to an estimate based on the average BMD. When applied to the assessment of Ct.Po within entire cortical bone cross-sections, the proposed BMD-based method had better accuracy than measurements performed with a conventional threshold-based approach.
\end{abstract}

\section{Introduction}

Cortical porosity (Ct.Po) is referred to cavities that permeate the extracellular mineralized matrix of cortical bone at several length scales, from millimeter-sized artery channels, to micro-scale (Haversian canals and resorption lacunae) and nano-scale (lacuno-canalicular network) pores. Throughout life, cortical bone is continuously remodeled, i.e., extracellular mineralized bone matrix is resorbed leaving remodeling cavities (also called basic multicellular units [BMUs]), which are subsequently refilled by new osteons including a central Haversian canal. These cylindrically shaped pores have a typical diameter between 25 and $200 \mu \mathrm{m}$ [1-3], contain blood vessels and are responsible for the major part of intracortical porosity [4]. The balance between bone tissue resorption and synthesis, which determines the porosity level, is affected by multiple factors, including age, sex, body size [5] and bone pathologies [6]. An alteration of this balance in adulthood (i.e., bone resorption rate exceeding bone formation rate) may lead to higher Ct.Po levels as a result of an increase in the number or size of pore, or a combination thereof.

Recent clinical studies using high-resolution peripheral quantitative computed tomography (HR-pQCT) associated cortical bone porosity increase with a higher incidence of fragility fractures of the distal radius $[7,8]$. These observations are in agreement with elastic and plastic theories of bone tissue, i.e., an increased porosity leads to decreased elastic properties [9-11] and fracture resistance [12]. In particular, researchers suggested that pores affect the fracture toughness of cortical bone by acting as stress concentrators [12,13]. With respect to this, not only the average porosity level but also the local distribution of pores

\footnotetext{
* Corresponding author.

E-mail address: kay.raum@charite.de (K. Raum).
} 
plays an important role. Indeed, the occurrence of local regions of high porosity caused by the accumulation of resorption cavities within the cortical shell reduces the strength of the femoral neck $[14,15]$. Due to the small size of Haversian canals, a direct in vivo assessment of Ct.Po with current medical imaging devices remains impossible.

Since its introduction, high-resolution peripheral quantitative computed tomography (HR-pQCT) is increasingly applied for the characterization of cortical porosity in vivo at peripheral sites of the skeleton (i.e., distal radius and tibia) $[16,17]$. The potential of HRpQCT-based Ct.Po measurements for fracture risk discrimination has been suggested recently [18]. These methods rely on the possibility to segment the spatially resolved cortical pore structure. However, such approaches are able to detect only pores that are larger than the spatial resolution limit, leading to a systematic underestimation of Ct.Po [19]. More recently, another strategy has been proposed, which utilizes bone mineral density (BMD) of HR-pQCT voxels as a surrogate measure of porosity [20]. This method is based on a two-phase composite material idealization of cortical bone, i.e., cortical bone is assumed to consist of a homogeneous extracellular bone matrix with a constant degree of mineralization and water-filled cavities. However, at the length scale of HR-pQCT voxels (i.e., $82 \mu \mathrm{m}$ and $61 \mu \mathrm{m}$ for first and second-generation systems, respectively), BMD is determined by the relative proportion of the void phase (i.e. pores) and by the mineral content of the extracellular bone matrix. Because of the low variability of the mineral content within mineralized bone tissue [21,22], Ct.Po has been suggested to be the major determinant of BMD. BMD voxels from HR-pQCT images convey information, which could be exploited for the direct assessment of cortical bone porosity. Recently, Jorgenson et al. [23] compared threshold and BMD-based approaches for the measurement of Ct.Po on $(5 \times 5 \times 5) \mathrm{mm}^{3}$ samples obtained from human tibiae, confirming a good agreement of both strategies by comparison with (gold standard) synchrotron radiation micro-computed tomography (SR $\mu \mathrm{CT})$.

In this context, we aimed at extending the BMD-based assessment of Ct.Po from an estimation of the sample average porosity to its local description (Study 1). To this end, we used registered second-generation HR-pQCT and 100-MHz scanning acoustic microscopy (SAM) images, which were obtained from femoral shaft samples of 20 human donors in vitro, to access local site matched BMD and Ct.Po. The characteristic error of the BMD-based measurement of Ct.Po was characterized for length scales from a few tens of microns up to the millimeter scale. We hypothesized that complementary information obtained from HR-pQCT scans could be used to further improve the Ct.Po estimation. We propose a multi-parameter model that utilizes local BMD combined with characteristics of the sample BMD histogram. Ct.Po estimates obtained with this method were compared with reference values from SAM as well as with conventional threshold-based Ct.Po estimates. Study 2 simulates the application of the proposed model to HR-pQCT images with lower resolution (mimicking typical in vivo scan protocols) whereas in Study 3 we demonstrate its validity for the analysis of $3 \mathrm{D}$ volumes.

\section{Materials and methods}

\subsection{Sample preparation}

Twenty pairs of proximal femur samples from human cadavers (7 male, 13 female, age: $69-94$ years, mean: $83.6 \pm 8.0$ years) were obtained from the Anatomy Institute of the Lübeck University in accordance with the German law "Gesetz über das Leichen-, Bestattungsund Friedhofswesen des Landes Schleswig-Holstein - Abschnitt II, §9 (Leichenöffnung, anatomisch)" from 04.02.2005. The bones were frozen at $-20^{\circ} \mathrm{C}$ immediately after dissection and were prepared for CT scanning and mechanical testing following an established protocol [24]. The proximal portion of each femur was extracted by cutting the bone at a distance of $80 \mathrm{~mm}$ distal from the middle point of the lesser trochanter and perpendicular to the functional axis of the femur shaft [25]. Soft tissue was removed from the femoral shaft, lesser trochanter and greater trochanter. The distal end (approx. $30 \mathrm{~mm}$ ) of the proximal shaft was embedded in polyurethane (SG 140/PUR 12, ebalta, Arundel, UK) (Fig. 1a).

\subsection{HR-pQCT scanning}

The samples were placed inside a desiccator, submerged in 1\% PBS solution, and exposed to partial vacuum (approx. $4 \mathrm{kPa}$ ) for $10 \mathrm{~min}$ right before scanning in order to remove air bubbles. The bones were then positioned inside a custom-made plastic chamber [26] (Fig. 1b) filled with $1 \%$ PBS, penicillin $(50 \mathrm{U} / \mathrm{ml})$ and streptomycin $(50 \mu \mathrm{g} / \mathrm{ml})$, and scanned with a second-generation HR-pQCT scanner (XtremeCT II; Scanco Medical AG, Brüttisellen, Switzerland) orienting the femur axis parallel to the z-axis of the scanner. Scanning settings were: $68 \mathrm{kVp} \mathrm{X}$ ray tube potential, $1470 \mu \mathrm{A} X$-ray tube current, $200 \mathrm{~ms}$ integration time for 3000 projections over $180^{\circ}$. The acquisition required up to 18 stacks; adjusting the field of view according to individual specimen length (min: $146 \mathrm{~mm}$; max: $182 \mathrm{~mm}$ ). Images were reconstructed as a $4608 \times 4608$ image matrix, yielding a $30.3 \mu \mathrm{m}$ isotropic voxel size. A 3D Gaussian filter ( $\sigma=1.1$ voxels, radius $=2.0$ voxels) was applied to the HR-pQCT image volume in order to remove high-frequency noise. Voxels integer values were converted to bone mineral density (BMD) with the scanner built-in calibration rule. According to the manufacturer's instructions, the validity of this rule was verified daily by scanning a calibration phantom with known densities (Scanco Medical AG, Brüttisellen, Switzerland). After scanning, the samples were frozen again at $-20^{\circ} \mathrm{C}$.

\subsection{Scanning acoustic microscopy (SAM)}

One 21-mm thick section (Fig. 1c) of the proximal shaft was extracted from each femur sample for a microelastic measurement by means of quantitative time-resolved SAM. Transversal cuts were performed with a band saw (EXACT GmbH, Remscheid, Germany) perpendicular to the shaft axis, $18 \mathrm{~mm}$ and $39 \mathrm{~mm}$ below the middle point of the lesser trochanter. Distances between cut planes and bottom of the sample were measured to determine the approximate position of the cross section within the HR-pQCT volume. The cross sections were washed 3 to 5 times in $1 \%$ PBS solution and approximately $5 \mathrm{~mm}$ of the distal side of each sample was embedded in acrylic resin (VariKleer ${ }^{\circledR}$, Buehler Ltd., Lake Bluff, IL). Proximal surfaces were then polished on a planar grinder (Phoenix 4000, Buehler Ltd., Illinois) at a constant speed of $50 \mathrm{rpm}$ and with decreasing grain size (ISO/FEPA grit: P80, P600, P1200, P2500 and P4000, Buehler Ltd., Illinois). Samples were maintained wet during surface preparation. After polishing, samples were washed and then submerged in 1\% PBS for vacuum degas inside a desiccator for at least $30 \mathrm{~min}$ to remove air bubbles from the cortical pores. Before scanning, surfaces were cleaned with a soft paintbrush.

The acoustic measurements were performed using a custom-built quantitative scanning acoustic microscope. Device and scanning procedure have been described in detail elsewhere [27,28]. A $100-\mathrm{MHz}$ spherically focused transducer (KSI $100 / 60^{\circ}$, KSI, Herborn, Germany) was used. The $-6 \mathrm{~dB}$ bandwidth of the confocal pulse echo was 84.4-100.7 MHz, and the $-6 \mathrm{~dB}$ depth of focus and lateral beam diameter in the focal plane were $139 \mu \mathrm{m}$ and $19.8 \mu \mathrm{m}$, respectively [29]. During measurements, samples were immersed in a temperature-controlled tank containing $25^{\circ} \mathrm{C}$ degassed $1 \%$ PBS. Images were acquired by moving the transducer along the $\mathrm{x}$-y-plane with a scan increment of $12 \mu \mathrm{m}$. The scan time was up to $5 \mathrm{~h}$. The recorded signals were filtered using a Chebyshev filter with cutoff frequencies of 5 and $200 \mathrm{MHz}$ and the amplitude of the reflected signal was determined as the maximum of the (Hilbert-transformed) envelope signal. A previously described procedure [30] for defocus correction and conversion to acoustic impedance values was applied. For this, a reference phantom consisting of 


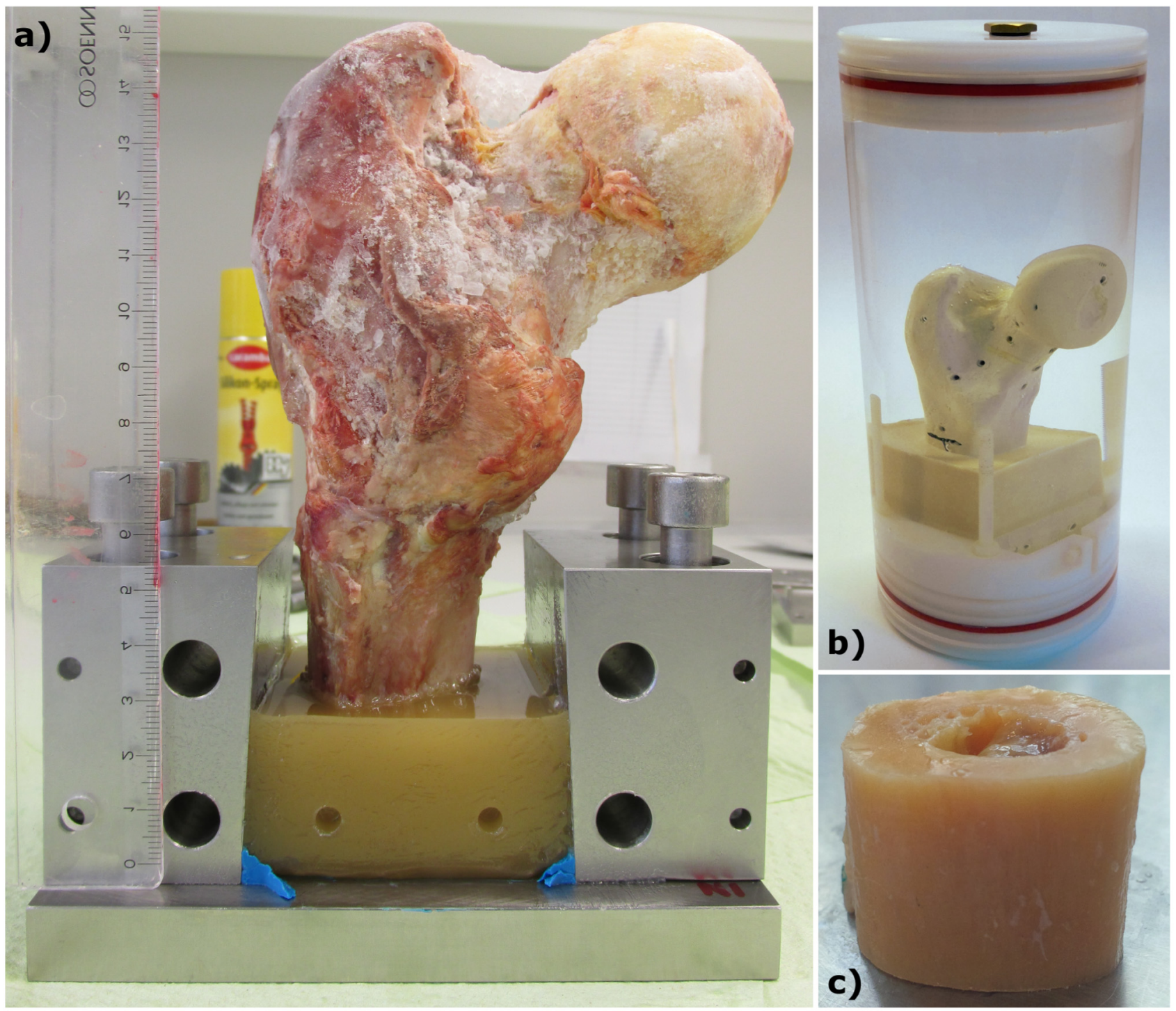

Fig. 1. (a) Preparation of a (left) proximal femur sample for HR-pQCT and SAM. The anatomical axis is drawn on the femoral shaft and a cut is realized perpendicular to this using a hand saw. A polyurethane embedding provided a holder for the HR-pQCT chamber (b). During preparation, the proximal side of the sample is wrapped with plastic bags containing dry ice (not shown here) in order to prevent the thawing of the hip. (c) $21 \mathrm{~mm}$ of the proximal shaft are extracted for SAM analysis.

titanium and polymethylmethacrylate (PMMA), i.e. two homogenous materials with known impedances was scanned before and after each bone sample measurement.

\subsection{Image analysis}

The image processing and data workflow to the individual substudies of this work are shown in Fig. 2. All analyses were performed in Matlab (R2017a, The Mathworks Inc., Natick, MA, USA).

\section{5. $3 D$ registration}

The 3D registration of the HR-pQCT data with the SAM cross-section was accomplished using a semi-automatic procedure described in the supplementary materials of this article. Briefly, the technique was based on an initial 2D registration step, performed in $x y$-planes of the HRpQCT volume to find the approximate longitudinal (z) position of the corresponding cross-section, followed by a second step, in which the 3D misalignment between the HR-pQCT and the SAM images was corrected. After 3D registration, HR-pQCT slices were resized by bicubic interpolation to match the pixel size of the SAM image. Plots of the 2D- correlation coefficient between the SAM image and single slices of the HR-pQCT stack were compared before and after 3D registration.

\subsection{Segmentation}

Segmentation of the SAM images was obtained by applying a previously described adaptive threshold [28]. For the HR-pQCT images, binary masks of the bone tissue were obtained using the Otsu's method [31]. Masks of the cortical bone compartment were automatically generated for HR-pQCT data using the algorithm proposed by Burghardt et al. [16]. This step was performed on both 2D slices registered to the SAM images and 3-mm thick ( $\sim 100$ slices) stacks extracted around the SAM plane. The masks were then morphologically eroded using a disk-shaped structuring element with a radius of $0.06 \mathrm{~mm}$ to compensate for the morphological closing applied by the cortical mask algorithm, which would lead to a slight overestimation of the periosteal surface. It should be noted that no manual correction of the endosteal boundary was applied. Posterior sites of muscle insertion corresponding to the linea aspera were manually cropped and excluded from the characterization of local properties, since it was not possible to separate the cortical bone compartment from the trabecular one for this site. 


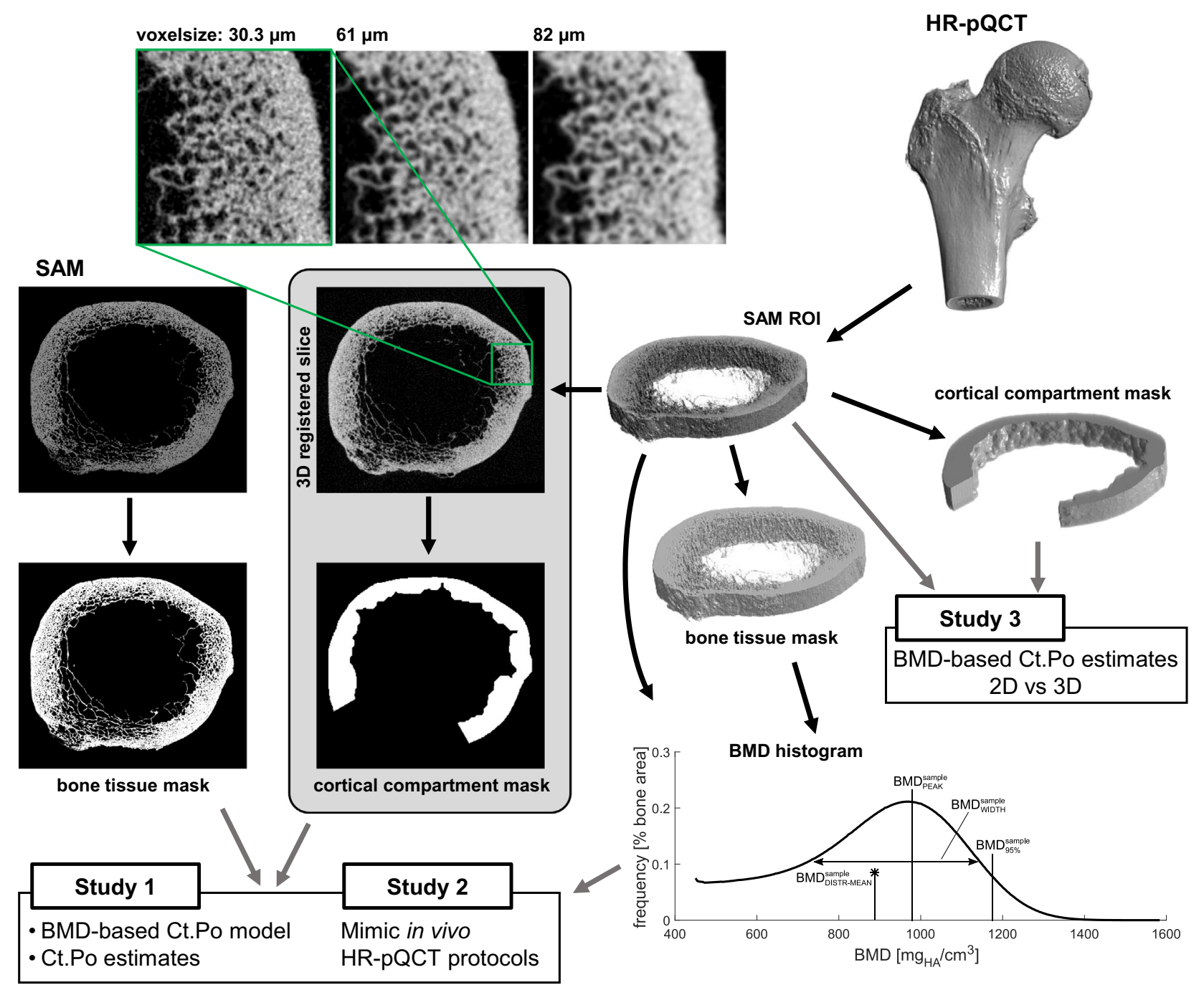

Fig. 2. Image processing steps for SAM (LEFT) and HR-pQCT (RIGHT) indicating the inputs of the different sub-studies.

\subsection{Study 1: modelling cortical bone porosity based on BMD}

Hereinafter, we use the superscripts ${ }^{\text {local }}$ and sample to distinguish variables assessed over local ROIs from those measured over entire samples.

\subsection{Model parameters and representative volume element (RVE) size}

An isotropic grid with increasing size $(0.060,0.084,0.132,0.204$, $0.324,0.444,0.540,0.660,0.756,0.90,1.044$, and $1.212 \mathrm{~mm}$ ) was overlaid to the registered SAM and HR-pQCT images (Fig. 3) and only those ROIs falling entirely within the cortical bone compartment were further analyzed. The lower grid size limit was chosen, as it represents the voxel size of 2nd generation HR-pQCT scanners for in vivo scans. For each grid ROI, the local average BMD (BMD $\left.D_{M E A N}^{\text {local }}\right)$ as well as the BMD inhomogeneity (expressed by means of the local BMD standard deviation $B M D_{S T D}^{\text {local }}$ ) were calculated from the HR-pQCT image, whereas $C t$. Polocal was extracted from the corresponding ROI of the SAM image. $C t$. Po ${ }^{\text {local }}$ was measured from the segmented images as the number of void pixels divided by the total number of ROI pixels. Correlations between $B M D_{M E A N}^{\text {local }}, B M D_{S T D}^{\text {local }}$ and Ct. Po ${ }^{\text {local }}$ were evaluated after pooling together the data for all samples.

In addition to the ROI-based BMD evaluation, histograms of the BMD distribution were derived, for each sample, within a sub-volume consisting of 100 slices $(\sim 3 \mathrm{~mm})$ centered at the SAM cross-section. The following parameters were extracted for each sample: $B M D_{95 \%}^{\text {sample }}$ (the BMD value corresponding to the 95th percentile of the BMD distribution), $B M D_{P E A K}^{\text {sample }}$ (the distribution peak frequency), $B M D_{D I S T R-M E A N}^{\text {sample }}$ and $B M D_{\text {WIDTH }}^{\text {sample }}$ (BMD distribution weighted mean and full width at half maximum, respectively) (Fig. 4).

A linear stepwise regression of all HR-pQCT parameters $\left(B M D_{M E A N}^{\text {local }}\right.$, $B M D_{S T D}^{\text {local }}, B M D_{D I S T R-M E A N}^{\text {sample }}, B M D_{P E A K}^{\text {sample }}, B M D_{W I D T H}^{\text {sample }}, B M D_{95 \%}^{\text {sample }}$ ) was used to model Ct. Polocal at each ROI size. Only ROIs with Ct. Polocal between 0 and $40 \%$ were considered to exclude from the analysis any region belonging to potentially trabecularized sites. After adding each significant parameter to the stepwise regression, porosity estimates were characterized in terms of their Root Mean Squared Error (RMSE), allowing the selection of the minimum number of explanatory variables for a Ct.Po model. A representative volume element (RVE) size was selected as the smallest ROI size providing an RMSE of the Ct. Polocal estimate below $3 \%$. This size was adopted for all further analyses.

\subsection{Ct.Po predictions}

After model derivation, Ct. Po ${ }^{\text {local }}$ predictions obtained using the RVE size were compared with the Ct. Polocal measured from SAM images. Ct.Po was also predicted for entire femoral cross-sections $\left(C t . P O^{\text {sample }}\right)$. In order to do this, $B M D_{M E A N}^{\text {sample }}$ and $B M D_{S T D}^{\text {sample }}$ were calculated over the cortical compartment. For comparison, thresholdbased measurements of $\mathrm{Ct}$. Po ${ }^{\text {sample }}$ were obtained for the same cortical 


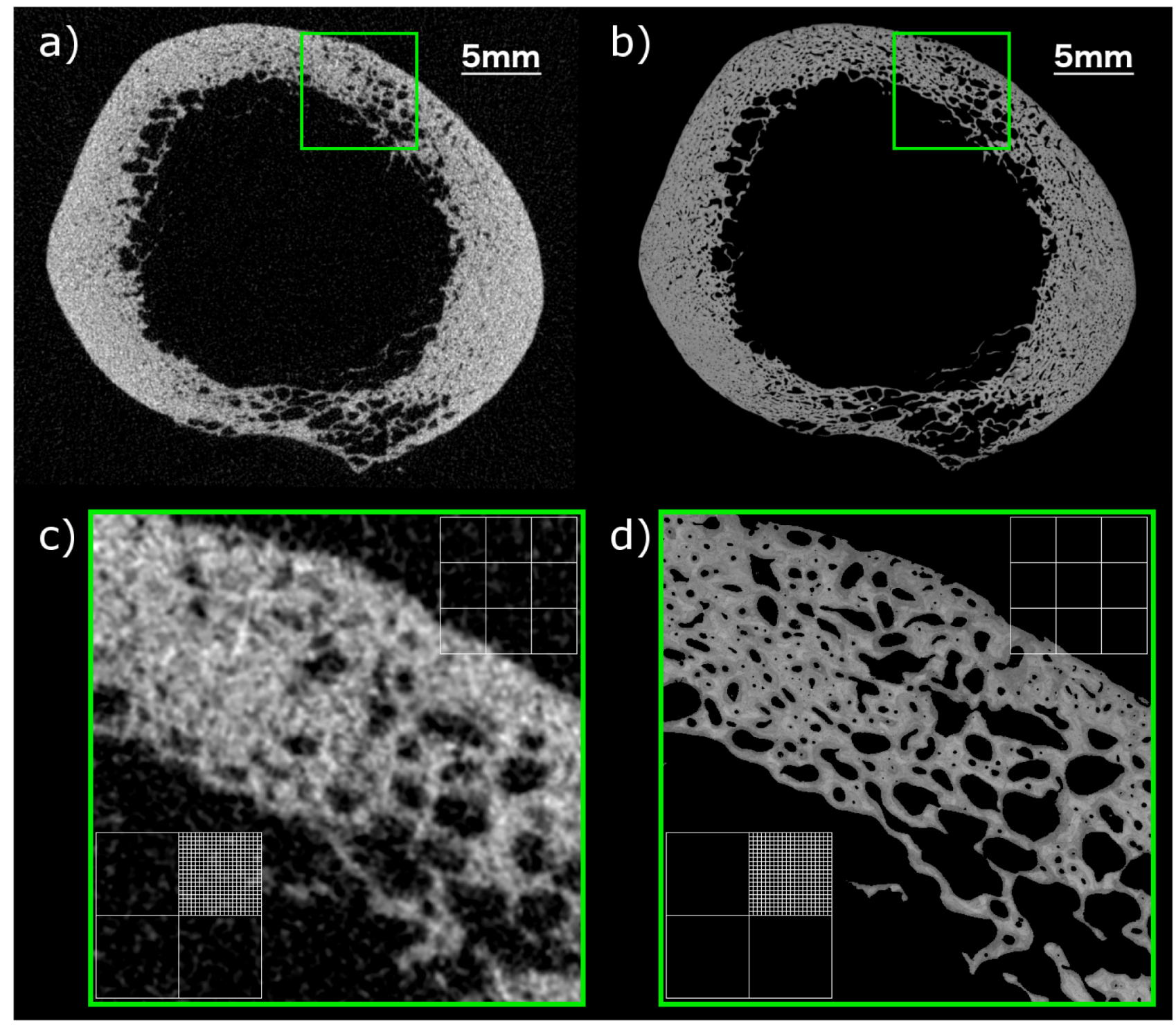

Fig. 3. Assessment of the local Ct.Po, BMD and acoustic impedance from 3D-registered HR-pQCT (a) and SAM (b) images. A detail of the cortex is shown in (c) (HRpQCT) and (d) (SAM) together with the largest and finest ROI sizes used for the assessment of local properties (bottom left corner of the detail images; large ROI size: $1.212 \mathrm{~mm}$, fine ROI size: $0.060 \mathrm{~mm})$. The RVE size selected for the analysis of correlation between local properties $(0.660 \mathrm{~mm})$ is shown on the top right corner of the detail images.

bone region by means of a previously described approach [16]. Both, BMD-model based and threshold-based Ct. Po sample values were compared with values measured with SAM.

\subsection{Study 2: conventional HR-pQCT resolutions}

In vivo HR-pQCT images were simulated from the original 3D registered volumes. First, a Gaussian filter was applied to mimic the point-spread function (PSF) of in vivo scan protocols of 1st and 2nd generation HR-pQCT scanners (i.e. 130 and $95 \mu \mathrm{m}$, respectively [32]). The volumes were then downsampled to the voxel size of the corresponding in vivo scan protocols (i.e., 82 and $61 \mu \mathrm{m}$ ) and $B M D_{M E A N}^{\text {local }}$,

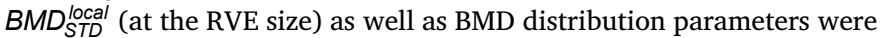
recalculated. The same procedure for the derivation of a porosity model and for the prediction of Ct. Po ${ }^{\text {local }}$ and Ct. Posample described in Section 2.7 was applied to the lower resolution data.

\subsection{Study 3: 3D Ct.Po estimates}

We investigated the agreement of 2D slice based Ct. Po sample predictions with those obtained from a 3-mm thick 3D cross-section extracted around the SAM cut plane. For predicting Ct. Po sample over a 3D volume, $B M D_{M E A N}^{\text {sample }}$ and $B M D_{S T D}^{\text {sample }}$ were extracted from the entire cortical bone volume.

\subsection{Statistical analysis}

Normality of the parameter distributions was tested using the Shapiro-Wilk test. Spearman's rank correlation coefficients $\rho$ were calculated to assess the relationship between BMD parameters and Ct.Po. Linear stepwise regressions were used for the model development. The following post-hoc leave-n-out test was performed for Ct.Po model cross-validation: 12 randomly selected samples (approx. 30\% of the data) were excluded for model derivation and the RMSE of the 


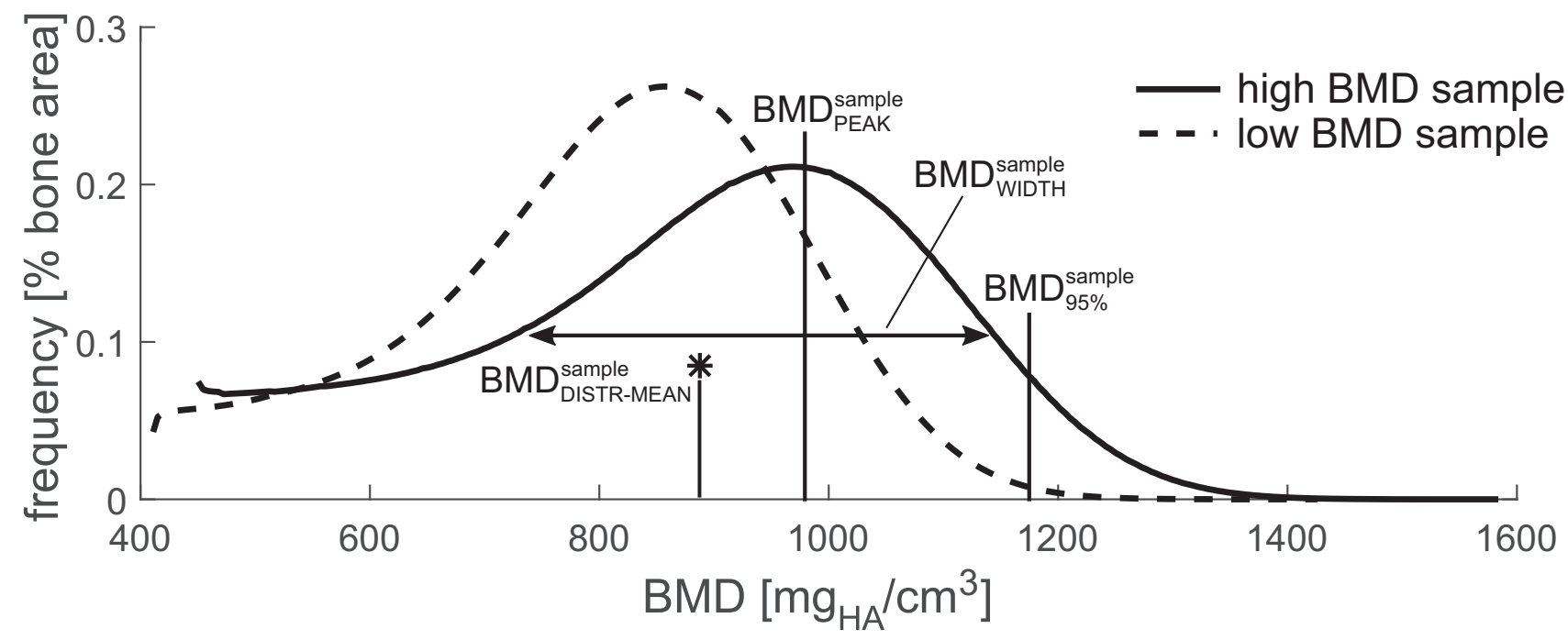

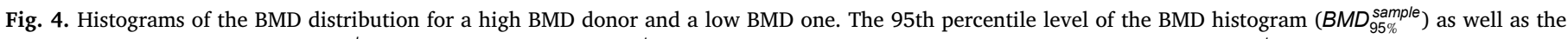

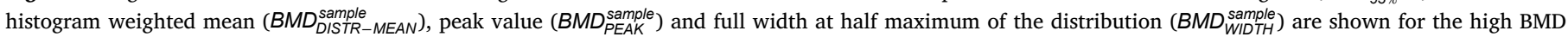
sample curve.

Ct. Polocal estimate of the left out samples was characterized; 1000 repetitions of the test were performed. Pearson linear regression analysis and Bland-Altmann plots [33] were used to compare the model predictions with the SAM-based values. Differences between properties measured on the left and right samples were tested using a Wilcoxon rank-sum test. A paired $t$-test (or Wilcoxon signed-rank test for all variables that did not follow a normal distribution) was used to assess sample "left versus right" differences as well as the agreement between Ct. Po sample estimates and reference Ct. Po sample from SAM. The significance level was set to $p=0.05$.

\section{Results}

\section{1. $3 D$ registration}

For 39 of the 40 evaluated samples, the 3D registration procedure was able to identify on the HR-pQCT volume a best fitting sectioning plane corresponding to the SAM image. The fitting algorithm converged, on average, after 114 trials. No convergence after a maximum number of 1000 trials was reached in one case. This exception could be explained by a severe misalignment (inclination) between the HR-pQCT slices plane and the cross-sectional plane scanned with SAM, invalidating the first $2 \mathrm{D}$ rigid registration step. This sample was excluded from further calculations. For the remaining 39 samples, the average maximum 2D correlation coefficients were 0.83 and 0.86 before and after 3D registration, respectively. An improvement of the correlation coefficient was observed for all samples after the 3D registration step (Fig. 5).

\subsection{Study 1}

\subsubsection{Ct.Po model}

Both $B M D_{M E A N}^{\text {local }}$ and $B M D_{S T D}^{\text {local }}$ were correlated with $C t$. Po local at all evaluated ROI sizes, with Spearman's $\rho$ ranging from 0.38 to 0.94 and from 0.21 to 0.91 for $B M D_{M E A N}^{\text {local }}$ and $B M D_{S T D}^{\text {local }}$, respectively (ROI size: $60 \mu \mathrm{m}, 2.1$ million evaluated ROIs to ROI size: $1.212 \mathrm{~mm}, 3294$ evaluated ROIs). Stepwise linear regression always included $B M D_{M E A N}^{\text {local }}$ as the first parameter, followed by $B M D_{S T D}^{\text {local }}$ and $B M D_{W I D T H}^{\text {sample }}$. Given the large number of observations, all variables had $p$-value smaller than 0.001. However, no further improvement of the model RMSE was observed after including $>3$ HR-pQCT variables (Fig. 6). We therefore restricted the number of model parameters to 3, yielding RMSE values of $8.89 \%$ and $2.57 \%$ for ROI sizes of $(60 \mu \mathrm{m})^{2}$ (not shown) and $(1.212 \mathrm{~mm})^{2}$, respectively (Fig. 6). The smallest ROI size reaching the criterion of RMSE $\leq 3 \%$ was $(660 \mu \mathrm{m})^{2}$, which was used for all further analyses. Under these conditions, the RMSE of Ct. Polocal dropped from

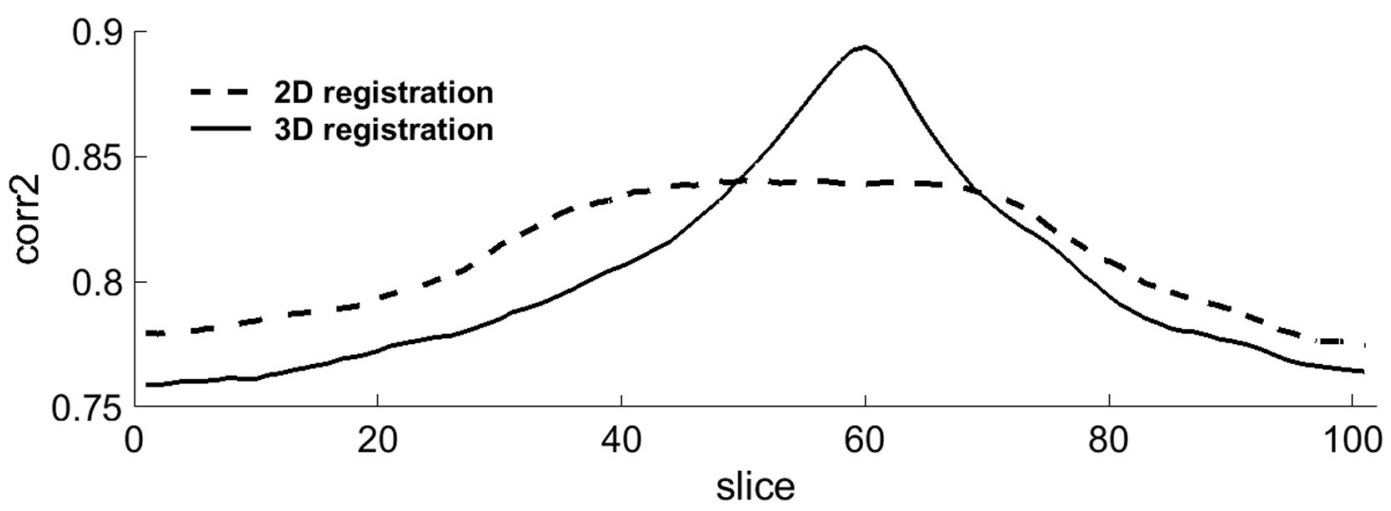

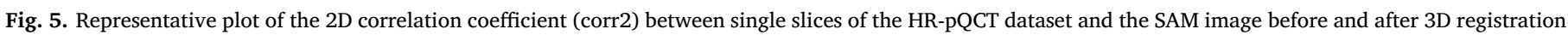

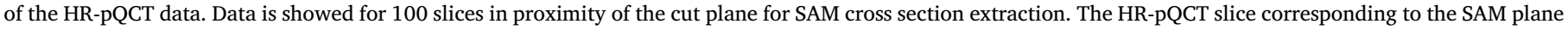
can be identified as the slice with maximum corr2 after 3D registration. 


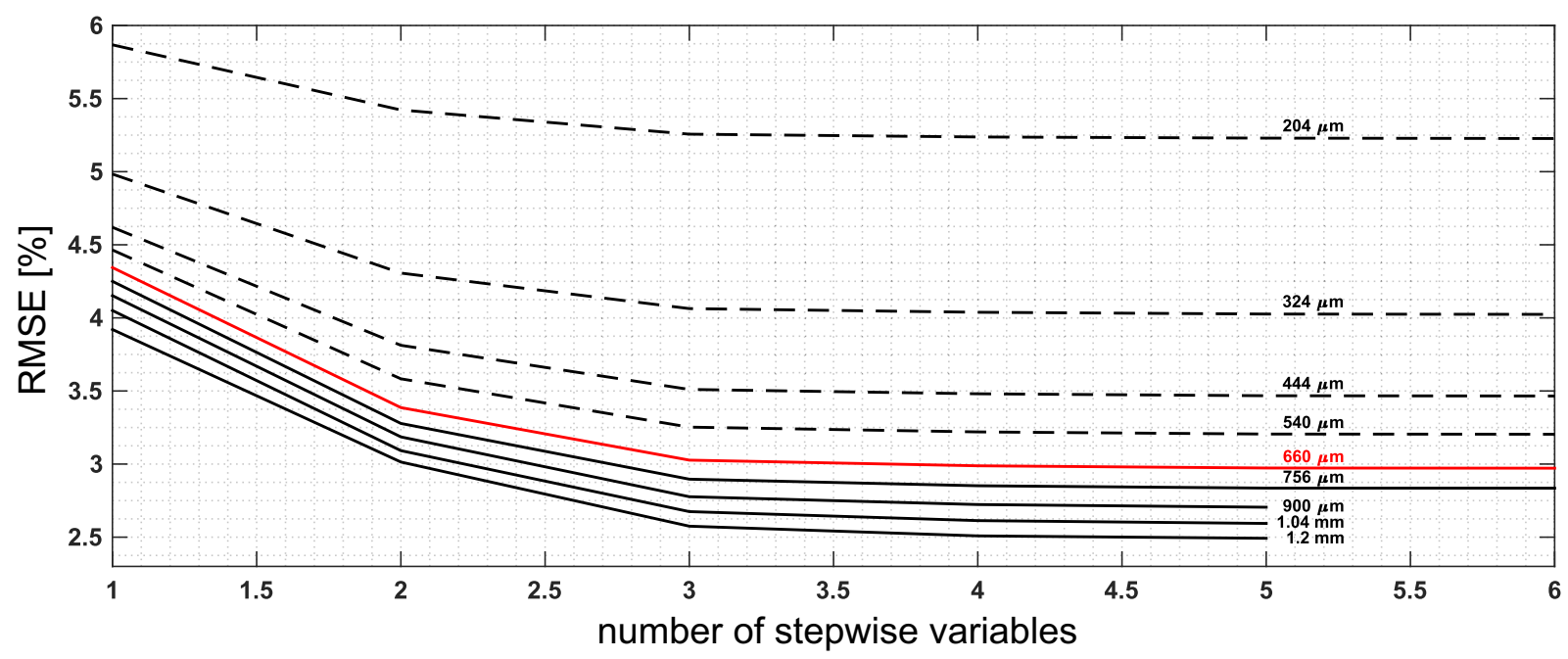

Fig. 6. Root Mean Squared Error of the Ct. Polocal prediction for increasing ROI size and number of explanatory HR-pQCT variables included in the stepwise Ct. Polocal model. Results obtained with ROI sizes of $0.060 \mathrm{~mm}, 0.084 \mathrm{~mm}$, and $0.132 \mathrm{~mm}$ are omitted for clarity.

$4.3 \%$ to $3.0 \%$ (30\% relative reduction) and 594 estimates were obtained, on average, per sample cross section (max: 825; min: 256 for the sample with the smallest cortical thickness).

The following model equation for Ct. Polocal was obtained:

$$
\begin{aligned}
& \text { Ct. } \text { Po }^{\text {local }}=36.79 \%-\left(0.0539 \mathrm{BMD}_{\mathrm{MEAN}}^{\text {local }}-0.0439 \mathrm{BMD}_{\mathrm{STD}}^{\text {local }}\right. \\
& \left.-0.0527 \mathrm{BMD}_{\mathrm{WIDTH}}^{\mathrm{sample}}\right) \frac{\%}{\mathrm{mg}_{\mathrm{HA}} / \mathrm{cm}^{3}}
\end{aligned}
$$

\subsubsection{Correlation of local and sample properties}

Local variables measured within $660 \times 660 \mu \mathrm{m}^{2}$ RVEs were pooled for all 39 samples: Ct. Po ${ }^{\text {local }}, B M D_{M E A N}^{\text {local }}$ and $B M D_{S T D}^{\text {local }}$ were in the range between 1.8 and $76.4 \%$ (CV: 68\%), 346-1121 $\mathrm{mg}_{\mathrm{HA}} / \mathrm{cm}^{3}$ (CV: $13 \%$ ) and $63-545 \mathrm{mg}_{\mathrm{HA}} / \mathrm{cm}^{3}$ (CV: $53 \%$ ), respectively, for a total number of 23.149 evaluated RVEs (Table 1). Approximately 5\% of all RVEs had Ct.Po $>40 \%$ and were discarded from the regression analyses. Both, $B M D_{M E A N}^{\text {local }}$ and $B M D_{S T D}^{\text {local }}$ were strongly correlated with Ct.Po ( $\rho=-0.87 ; p<0.001$ and $\rho=0.89 ; p<0.001$ ). When measured over entire sample cross-sections, the BMD inhomogeneity $\left(B M D_{S T D}^{\text {sample }}\right)$

Table 1

\begin{tabular}{|c|c|c|c|c|}
\hline $\begin{array}{l}\text { Local properties } \\
(N=23.149)\end{array}$ & Mean \pm SD & Range & rho & $p$ \\
\hline Ct. Polocal $[\%]$ & $16.7 \pm 11.3$ & $1.8 \div 76.4$ & & $\mathrm{~ns}^{\mathrm{a}}$ \\
\hline$B M D_{M E A N}^{\text {local }}\left[\mathrm{mg}_{\mathrm{HA}} / \mathrm{cm}^{3}\right]$ & $855.3 \pm 113.0$ & $346.1 \div 1121.0$ & -0.87 & $\mathrm{~ns}^{\mathrm{a}}$ \\
\hline$B M D_{S T D}^{\text {local }}\left[\mathrm{mg}_{\mathrm{HA}} / \mathrm{cm}^{3}\right]$ & $181.9 \pm 96.7$ & $63.1 \div 545.4$ & 0.89 & $0.02^{\mathrm{a}}$ \\
\hline \multicolumn{5}{|l|}{ Sample properties $(N=39)$} \\
\hline Ct. Po sample $[\%]$ & $12.0 \pm 3.6$ & $6.8 \div 21.0$ & & $\mathrm{~ns}^{\mathrm{b}}$ \\
\hline$B M D_{M E A N}^{\text {sample }}\left[\mathrm{mg}_{\mathrm{HA}} / \mathrm{cm}^{3}\right]$ & $898.8 \pm 36.6$ & $820.4 \div 973.6$ & -0.57 & ns \\
\hline$B M D_{S T D}^{\text {sample }}\left[\mathrm{mg}_{\mathrm{HA}} / \mathrm{cm}^{3}\right]$ & $152.1 \pm 16.5$ & $125.0 \div 190.2$ & 0.80 & $\mathrm{~ns}^{\mathrm{b}}$ \\
\hline$B M D_{W I D T H}^{\text {sample }}\left[\mathrm{mg}_{\mathrm{HA}} / \mathrm{cm}^{3}\right]$ & $335.5 \pm 29.9$ & $298.6 \div 422.7$ & 0.70 & $\mathrm{~ns}^{\mathrm{b}}$ \\
\hline $\begin{array}{l}B M D_{D I S T R-M E A N}^{\text {sample }}\left[\mathrm{mg}_{\mathrm{HA}} /\right. \\
\left.\mathrm{cm}^{3}\right]\end{array}$ & $867.4 \pm 39.8$ & $789.8 \div 949.4$ & -0.71 & ns \\
\hline$B M D_{P E A K}^{\text {sample }}\left[\mathrm{mg}_{\mathrm{HA}} / \mathrm{cm}^{3}\right]$ & $927.7 \pm 33.0$ & $857.0 \div 990.5$ & -0.39 & ns \\
\hline$B M D_{95 \%}^{\text {sample }}\left[\mathrm{mg}_{\mathrm{HA}} / \mathrm{cm}^{3}\right]$ & $1132.5 \pm 40.5$ & $1054.4 \div 1224.7$ & -0.48 & ns \\
\hline
\end{tabular}

Local and sample intracortical porosity and BMD parameters.

Mean value, standard deviation (SD); minimum and maximum values; correlation with Ct.Po (Spearman's rho); significance of the paired sample $t$-test (p) for the comparison "left versus right".

${ }^{\text {a }}$ Wilcoxon rank-sum test.

b Wilcoxon signed rank test. was a better predictor for Ct. Po sample than $B M D_{M E A N}^{\text {sample }}$ (Spearman's $\rho=0.80 ; \mathrm{p}<0.001$ and $\rho=-0.57 ; \mathrm{p}<0.001$, respectively). No difference between left and right populations was observed for all sample variables. Only $B M D_{S T D}^{\text {local }}$ was different between left and right.

\subsubsection{Ct.Po estimates}

Ct. Po local estimates (RVE size: $(660 \mu \mathrm{m})^{2}, 23.149$ ROIs evaluated) obtained with Eq. (1) showed excellent agreement with the local Ct. Po local measured by SAM, providing a correlation coefficient of $\mathrm{R}^{2}=0.91(\mathrm{p}<0.001)$ and root mean squared error of the estimate RMSE $=3.4 \%$ (Fig. 7).

Similarly, Ct. Po sample estimates for 2D HR-pQCT slices were in very good agreement with $C t$. Po sample obtained from the corresponding registered SAM images $\left(n=39, \mathrm{p}<0.001, \mathrm{R}^{2}=0.80\right.$, Fig. 8a). In contrast, the threshold-based approach had a lower correlation coefficient $\left(\mathrm{R}^{2}=0.77, p<0.001\right)$ and was affected by a measurement bias that was highly dependent on the $C t$. Posample level (Fig. 8c). Both methods significantly underestimated Ct. Po sample $(p<0.001)$ but the deviation of the threshold-based approach remained much larger (mean difference: $-10.44 \%$ versus $-0.91 \%$ for the BMD-based method, Fig. $8 \mathrm{~b}$ and c).

\subsubsection{Model cross-validation}

Twelve out of 39 samples were left out for Ct. Polocal model crossvalidation. Ct. Polocal estimates obtained for the left out data points had an RMSE of $3.51 \pm 0.22 \%$ (min: $2.88 \%$; max: $4.32 \%$ ), which was only slightly larger than the $3.4 \%$ error obtained with the entire sample set (Fig. 7a).

\subsection{Study 2: simulated in vivo resolution $H R-p Q C T$}

A simulated degradation of the image resolution affected the calculation of the BMD (local as well as sample) inhomogeneity as well as all BMD distribution parameters (Table 2). Nevertheless, the correlation between BMD parameters and porosity remained essentially unchanged.

The error of the Ct. Polocal estimate obtained from simulated in vivo images was only $0.1 \%$ and $0.2 \%$ larger ( $3 \%$ and $6 \%$ relative increase), respectively for 2 nd and 1 st generation HR-pQCT, if compared to native resolution (Table 3). The effect on Ct. Po sample estimates was stronger: $9 \%$ and $18 \%$ relative increase of the RMSE compared to results obtained with $30.3 \mu \mathrm{m}$ voxels. 
a)

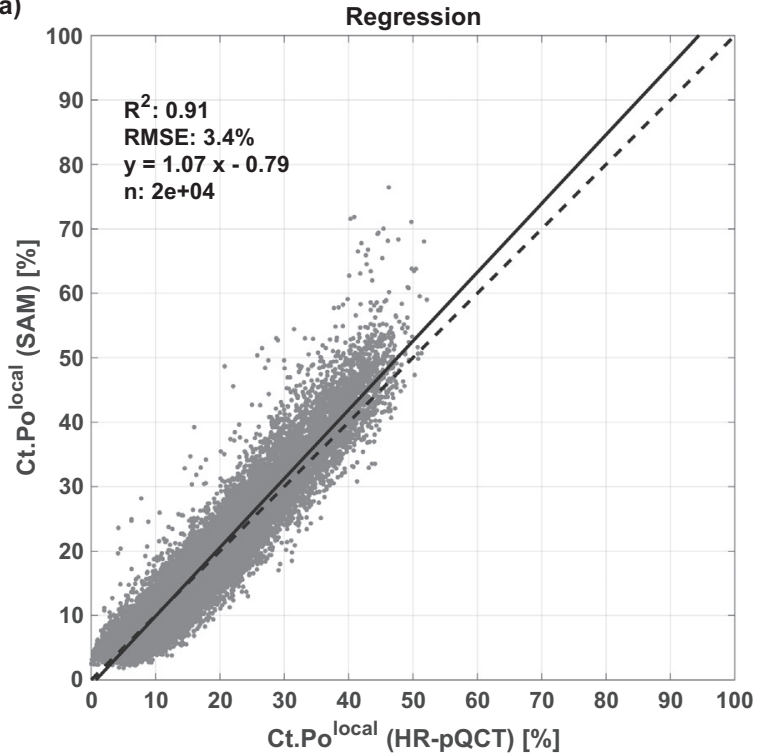

b)

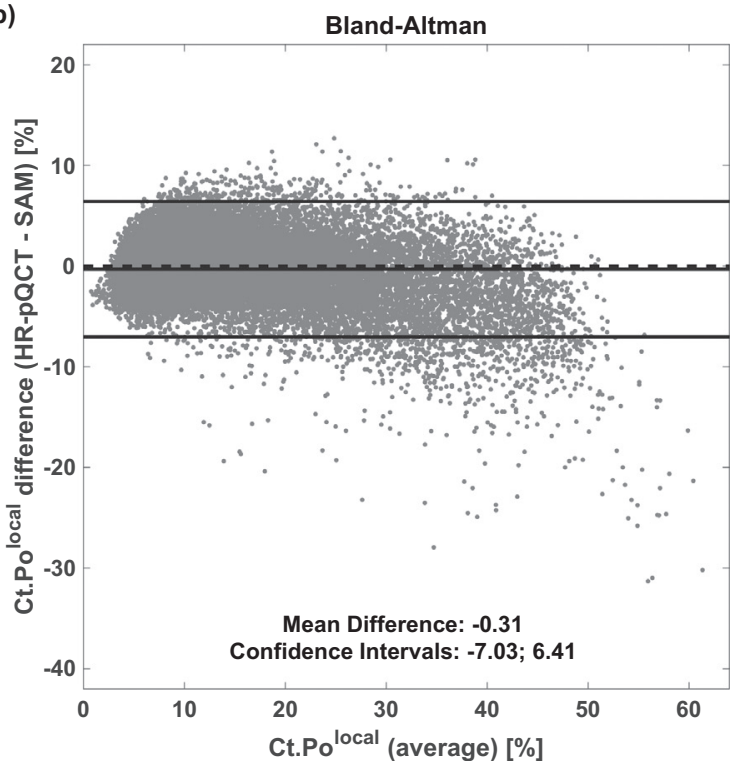

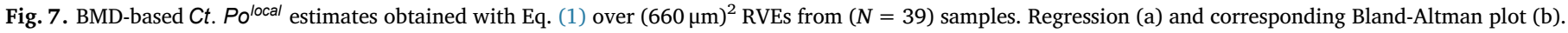

\subsection{Study 3: Ct. Po sample prediction from $3 D$ volumes}

BMD-based Ct. Po sample predictions obtained from 2D cross-sections were not significantly different from predictions obtained from 3-mm thick regions extracted around the SAM cross-section ( $p=0.60$, Fig. 9).

\section{Discussion}

Within this ex vivo study on human proximal femur samples, we used registered HR-pQCT and SAM images to investigate the local association between volumetric BMD and porosity in cortical bone. At a spatial length scale of $(660 \mu \mathrm{m})^{2}$, both the BMD inhomogeneity (assessed as the standard deviation of BMD within the investigated region) as well as the average BMD were strongly correlated with the local Ct.Po (Spearman's $\rho=0.89$ and -0.87 , respectively) throughout the cortex of 20 human donors. When sample properties were calculated considering entire cortical sections, the inhomogeneity of BMD alone became the better predictor of $C t$. Po ${ }^{\text {sample }}$ (Spearman's $\rho=0.80$ ). The strong correlation between BMD as assessed by QCT and bone porosity was already reported for trabecular [26,34] as well as for cortical bone $[17,34,35]$. These studies have investigated bone regions with dimensions of a few millimeter or more. Our study on HR-pQCT images confirms the BMD-Ct.Po correlation also for sub-millimeter length scales, suggesting that the information contained within the HR-pQCT voxels may be exploited for an accurate estimate of the local porosity.

\subsection{Study 1: BMD-based Ct.Po assessment}

We propose the assessment of $C t$. Po ${ }^{\text {local }}$ based on the local BMD as well as on the distribution of BMD throughout the entire examined cortical bone tissue.

When added to the porosity model, the BMD inhomogeneity $\left(B M D_{S T D}^{\text {local }}\right)$ together with $B M D_{W I D T H}^{\text {sample }}$, a parameter derived from the sample BMD distribution histogram, provided a relative reduction of a)

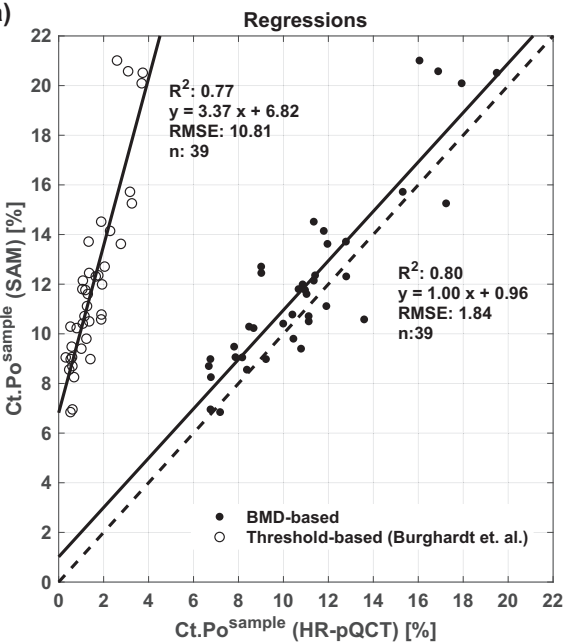

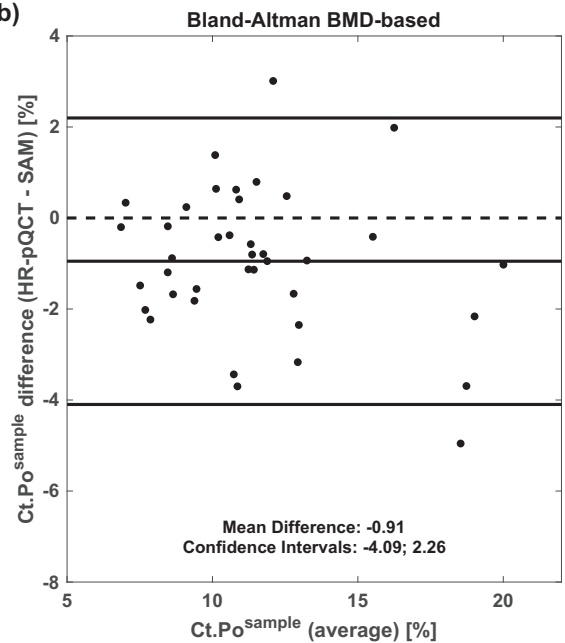

c)

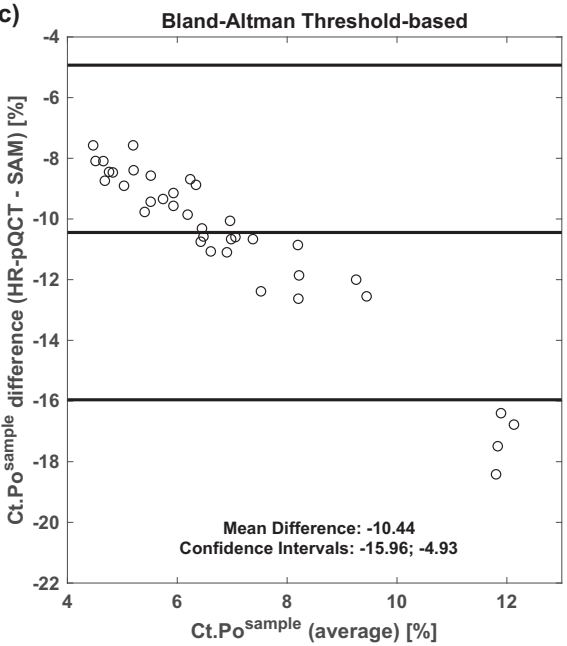

Fig. 8. Comparison of BMD-based Ct. Posample estimates obtained for entire proximal femur cross-sections by means of the proposed model with Ct. Po ${ }^{\text {sample }}$ measurements realized on the same HR-pQCT cross section using a threshold-based approach. Linear regression analysis (a) and Bland-Altman plots (b and c) of the comparison of both HR-pQCT techniques with the reference (12- $\mu \mathrm{m}$ pixel size SAM). Mean measurement difference and its confidence intervals are shown with horizontal lines in (b) and (c). ( $N=39$ cadaveric proximal femur samples from human donors). 
Table 2

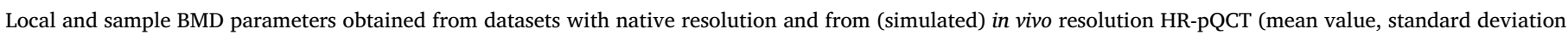
(SD) and Spearman's rho of the correlation with Ct.Po).

\begin{tabular}{|c|c|c|c|c|c|c|}
\hline \multirow[t]{2}{*}{ Local properties $(N=23.149)$} & \multicolumn{2}{|c|}{ 2nd generation; in vitro } & \multicolumn{2}{|c|}{ 2nd generation; in vivo } & \multicolumn{2}{|c|}{ 1st generation; in vivo } \\
\hline & Mean \pm SD & rho & Mean \pm SD & rho & Mean $\pm S D$ & rho \\
\hline$B M D_{M E A N}^{l o c a l}\left[\mathrm{mg}_{\mathrm{HA}} / \mathrm{cm}^{3}\right]$ & $855.3 \pm 113.0$ & -0.87 & $854.8 \pm 113.0$ & -0.88 & $854.4 \pm 113.0$ & -0.88 \\
\hline$B M D_{S T D}^{\text {local }}\left[\mathrm{mg}_{\mathrm{HA}} / \mathrm{cm}^{3}\right]$ & $181.9 \pm 96.7$ & 0.89 & $149.8 \pm 104.5$ & 0.89 & $137.6 \pm 106.4$ & 0.88 \\
\hline \multicolumn{7}{|l|}{ Sample properties $(N=39)$} \\
\hline$B M D_{M E A N}^{\text {sample }}\left[\mathrm{mg}_{\mathrm{HA}} / \mathrm{cm}^{3}\right]$ & $898.8 \pm 36.6$ & -0.57 & $896.5 \pm 36.7$ & -0.58 & $894.9 \pm 36.7$ & -0.58 \\
\hline$B M D_{S T D}^{\text {sample }}\left[\mathrm{mg}_{\mathrm{HA}} / \mathrm{cm}^{3}\right]$ & $152.1 \pm 16.5$ & 0.80 & $123.6 \pm 16.7$ & 0.83 & $114.6 \pm 16.0$ & 0.83 \\
\hline$B M D_{\text {WIDTH }}^{\text {sample }}\left[\mathrm{mg}_{\mathrm{HA}} / \mathrm{cm}^{3}\right]$ & $335.5 \pm 29.9$ & 0.70 & $233.3 \pm 35.1$ & 0.64 & $213.6 \pm 38.8$ & 0.65 \\
\hline 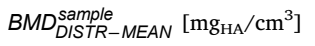 & $867.4 \pm 39.8$ & -0.71 & $858.0 \pm 40.9$ & -0.73 & $851.7 \pm 42.1$ & -0.76 \\
\hline$B M D_{P E A K}^{\text {sample }}\left[\mathrm{mg}_{\mathrm{HA}} / \mathrm{cm}^{3}\right]$ & $927.7 \pm 33.0$ & -0.39 & $934.2 \pm 35.4$ & -0.33 & $934.6 \pm 35.3$ & ns \\
\hline$B M D_{95 \%}^{\text {sample }}\left[\mathrm{mg}_{\mathrm{HA}} / \mathrm{cm}^{3}\right]$ & $1132.5 \pm 40.5$ & -0.48 & $1059.1 \pm 35.8$ & -0.41 & $1040.6 \pm 34.4$ & -0.37 \\
\hline
\end{tabular}

Table 3

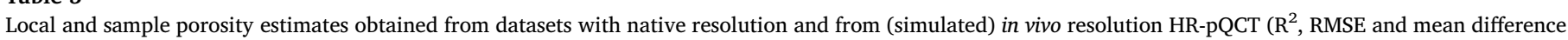
(MD) of the comparison with reference SAM Ct.Po).

\begin{tabular}{|c|c|c|c|c|c|}
\hline Ct. Polocal & $\begin{array}{l}\text { Resolution } \\
{[\mu \mathrm{m}]}\end{array}$ & $\begin{array}{l}\text { Voxel size } \\
{[\mu \mathrm{m}]}\end{array}$ & $\mathrm{R}^{2}$ & $\begin{array}{l}\text { RMSE } \\
{[\%]}\end{array}$ & $\mathrm{MD}[\%]$ \\
\hline 2nd generation; in vitro & 55.9 & 30.3 & 0.91 & 3.4 & -0.3 \\
\hline 2nd generation; in vivo & 95.0 & 61.0 & 0.91 & 3.5 & -0.3 \\
\hline 1st generation; in vivo & 130.0 & 82.0 & 0.90 & 3.6 & -0.3 \\
\hline \multicolumn{6}{|l|}{ Ct. Po sample } \\
\hline 2nd generation; in vitro & 55.9 & 30.3 & 0.80 & 1.8 & 0.8 \\
\hline 2nd generation; in vivo & 95.0 & 61.0 & 0.77 & 2.0 & 1.1 \\
\hline 1st generation; in vivo & 130.0 & 82.0 & 0.74 & 2.2 & 1.3 \\
\hline \multicolumn{6}{|l|}{ Model coefficients } \\
\hline 2nd generation; in vitro & \multicolumn{5}{|c|}{ Ct. $P o^{\text {local }}=36.79 \%-\left(0.0539 B M D_{\text {MEAN }}^{\text {local }}-0.0439 B M D_{S T D}^{\text {local }}-0.0527 B M D_{W I D T H}^{\text {sample }}\right) \frac{\%}{\mathrm{mg}_{\mathrm{HA}} / \mathrm{cm}^{3}}$} \\
\hline 2nd generation; in vivo & \multicolumn{5}{|c|}{ Ct. Polocal $=45.24 \%-\left(0.0525 B M D_{M E A N}^{\text {local }}-0.0421 B M D_{S T D}^{\text {local }}-0.0416 B M D_{W I D T H}^{\text {sample }}\right) \frac{\%}{\mathrm{mg}_{\mathrm{HA}} / \mathrm{cm}^{3}}$} \\
\hline 1st generation; in vivo & \multicolumn{5}{|c|}{ Ct. $P O^{\text {local }}=49.48 \%-\left(0.0542 B M D_{M E A N}^{\text {local }}-0.0397 B M D_{S T D}^{\text {local }}-0.0359 B M D_{W I D T H}^{\text {sample }}\right) \frac{\%}{\mathrm{mg}_{\mathrm{HA}} / \mathrm{cm}^{3}}$} \\
\hline
\end{tabular}

the Ct. Polocal estimate RMSE of 30\% compared with a model based on $B M D_{M E A N}^{\text {local }}$ alone. It should be noted that the model of Eq. (1) was obtained experimentally with no a priori assumption regarding the attenuation or BMD level of fully mineralized bone and provided Ct. Po ${ }^{\text {local }}$ estimates with an RMSE of 3.4\% for sub-millimeter regions of compact cortical bone (Ct. Polocal $<40 \%)$.

A variety of texture indexes was proposed for the structural characterization of bone from CT images, which were not investigated here. The potential of fractal measurements such as lacunarity and fractal signature [36] or variogram approaches like the Trabecular Bone Score (TBS) [37] was demonstrated for trabecular bone. Recently, Lowitz et al. applied BMD inhomogeneity measurements (together with four other texture parameters) to HR-pQCT images of (trabecular and) cortical bone ROIs of human knee joints [38]. Our study makes use of very limited texture information but shows how this can be utilized for the measurement of porosity also in cortical bone.

We applied the derived model to obtain Ct. Po sample estimates for entire cortical bone cross-section and compared these results with threshold-based Ct. Po sample estimates. In agreement with the results of other studies [17,20,23], a threshold-based approach underestimates Ct. Po sample due to its intrinsic inability to detect pores with characteristic sizes below the scanner resolution. Our data confirms this finding also for second generation HR-pQCT images obtained at $30.3 \mu \mathrm{m}$ voxel size.

As already reported by Zebaze et al. [20], the bias of the thresholdbased measurement was dependent on the Ct. Po ${ }^{\text {sample }}$ level (see Bland-
Altman plot of Fig. 8c). In the study of Jorgenson et al. [23], a similar trend is visible for Ct.Po up to $20 \%$. For larger values (up to $50 \%$ ), the bias became independent of Ct.Po.

\subsection{Study 2: towards in vivo HR-pQCT}

Our HR-pQCT images were acquired using a voxel size $(30.3 \mu \mathrm{m})$ which is only available ex vivo. This poses a question concerning the translation of our findings for in vivo HR-pQCT applications (for comparison, the voxel size for in vivo measurements are 82 and $61 \mu \mathrm{m}$ for first and second-generation HR-pQCT systems, respectively). Primarily, the level of porosity information conveyed by BMD voxels needs to be confirmed also for lower resolution HR-pQCT protocols. For this study, we simulated second $(61 \mu \mathrm{m})$ to first $(82 \mu \mathrm{m})$ generation HR-pQCT in vivo voxels by low-pass filtering and downsampling the $30.3 \mu \mathrm{m}$ voxels obtained with the ex vivo scanning protocol of a second-generation HRpQCT. BMD distribution histograms are flattened and constantly shifted towards lower BMD levels as the voxel size increases. Nevertheless, BMD inhomogeneity and BMD distribution information remained available also for the simulated in vivo images. Particularly, the correlation coefficient between porosity and the BMD inhomogeneity remained as high as 0.88 and 0.83 for local and sample measurements, respectively, suggesting that this method may also be applicable for in vivo measurements in patients. The coefficients of the porosity model were different for the three investigated image resolutions (Table 3), confirming that specific calibration rules should be established with 
a)

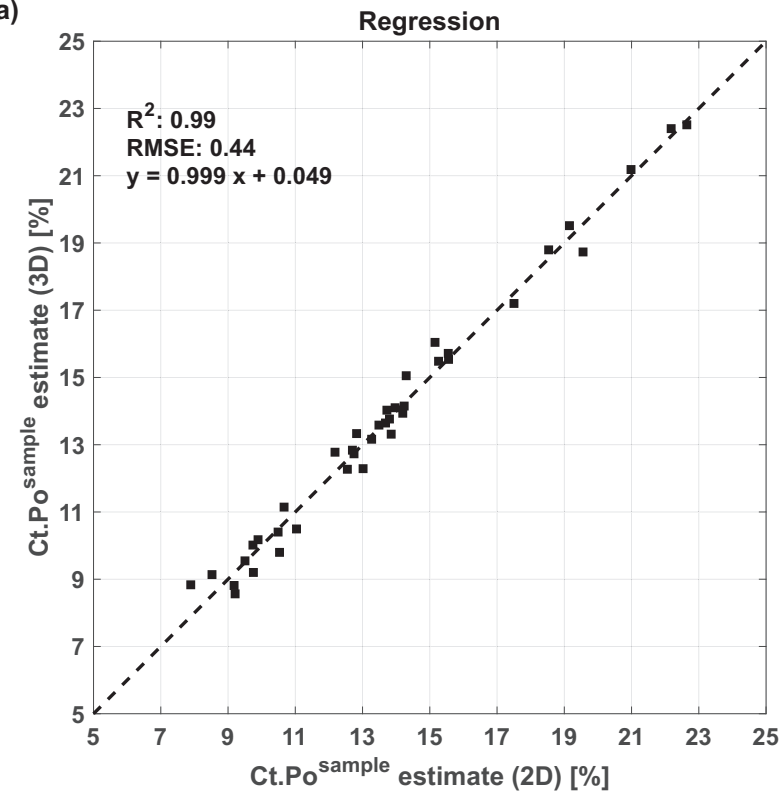

b)

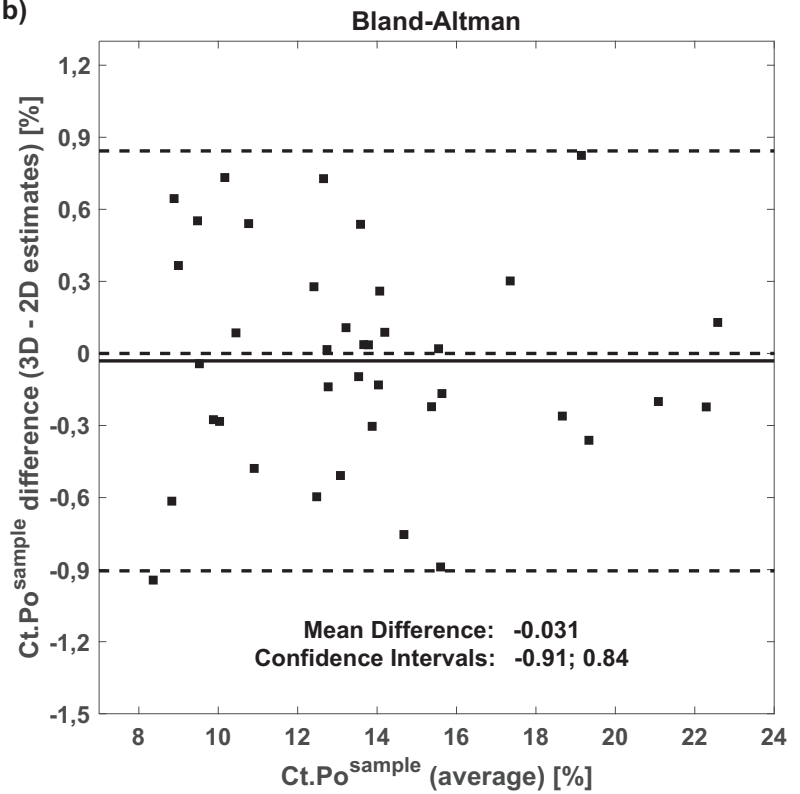

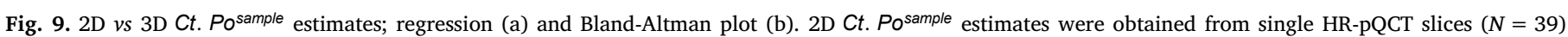

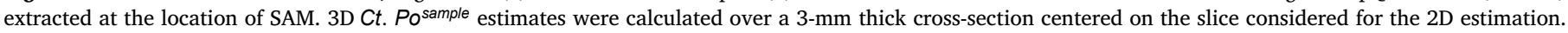

respects to scanner, measurement site, and system settings.

Besides image quality, the effect of artifacts such as image noise and beam hardening changes with scan resolution and source voltage as well as within different families of devices. The signal-to-noise ratio is also affected by the presence of soft tissues, which could be disregarded in our experiment. In a recent multi-site investigation, Burghardt et al. [39] concluded on the good agreement of bone density measurements performed with different HR-pQCT scanners. BMD assessments were less affected by intra-site variability compared to structural (i.e., Ct.Po) parameters obtained by conventional segmentation. In agreement with this finding, our results further support the reliability of a porosity calibration rule for HR-pQCT scanners based on BMD distribution parameters.

\subsection{Study 3: validity of a $3 D \mathrm{Ct}$. Po ${ }^{\text {sample }}$ estimation}

Previous comparative studies made use of 3D synchrotron radiation $\mu \mathrm{CT}(\mathrm{SR} \mu \mathrm{CT})$ as reference for $C t$. Po sample $[17,23]$. SR $\mu \mathrm{CT}$ provides 3D images at the necessary resolution. However, the field of view is limited, which restricts the analysis to small tissue regions. In our study, we obtained Ct. Po $\mathrm{P}^{\text {sample }}$ by means of SAM, which allowed us to measure porosity over entire femoral shaft cross-sections, but on 2D planes.

$100-\mathrm{MHz}$ SAM provides a spatial resolution similar to that obtained by SR $\mu C T$ at $10-\mu \mathrm{m}$ voxel size $[29,40]$. In diaphyseal cortical bones, the Haversian canals are predominantly orientated parallel to the long bone axis and their average length is $4 \mathrm{~mm}$ [41]. Due to this translational symmetry, the porosity values derived from a single cross-section can be assumed representative also for adjacent cross-sectional stacks. To verify that our procedure for the BMD-based $C t$. Po sample estimate is also valid for 3D HR-pQCT cross-sections (for which reference data was not available) we compared single 2D cross-sections with those assessed in an adjacent 3-mm thick volume. The results support the generic applicability of the proposed model.

\subsection{Perspective}

A BMD-based measurement of Ct. Polocal offers several advantages. First, the scanner ability to resolve and threshold single cortical pores do not limit the Ct. Po ${ }^{\text {local }}$ estimation. This allows to minimize partial volume effects and to take into account the contribution of pores with characteristic diameters below the resolution limit. It should be noted, however, that the reported model does not consider pores smaller than the resolution limit of the SAM image, e.g. osteocytes and their canaliculi. The local character of the proposed porosity model allows not only the estimation of a patient-specific mean cortical bone porosity, but also the 3D mapping of local cortical porosity within the scanned bone region. This can be used for the detection of local regions with altered pore morphology, e.g., regions affected by a higher bone resorption rate and subsequent bone loss, impaired by decreased elastic properties [11] and bone fracture resistance $[14,15,42]$.

Techniques based on the 3D mapping of the bone tissue mechanical properties from BMD voxels have been proposed and validated for Finite Element analyses of the mechanical competence of long bones $[43,44]$. Particularly, the relationship of bone tissue porosity with both elastic and failure properties have been elucidated [9-12, 45]. While the macroscopic mechanical behavior of cortical and trabecular tissue has been suggested to depend similarly on the bone volume fraction [44], calibration rules for the local mapping of the volume fraction from BMD have been established only for trabecular tissue [26]. Our work extends this approach also to human cortical bone tissue.

Another field of application of the Ct. Polocal mapping is the combination with in vivo bone quantitative ultrasound (QUS). The transmission of acoustic waves through and along the cortical bone shell can be used to infer structural (e.g., Ct.Po, Ct.Th) and material (e.g., extracellular matrix mineralization and stiffness) properties of cortical bone $[46,47]$. However, the relative contributions of structural and material properties to the measured sound propagation characteristics remain challenging to discern [46]. QUS devices are portable, use non-ionizing radiation, and are increasingly applied in clinical studies $[48,49]$ at distal sites of the skeleton (e.g., radius and tibia), which represent the same imaging target of HR-pQCT. With this respect, 3D descriptions of the local cortical porosity obtained from HR-pQCT could be combined with site-matched experimental measurements and numerical simulations of ultrasound propagation in long bones, to help elucidating the interplay between ultrasound and the cortical microstructure. 


\section{Conclusions}

BMD measurements obtained by HR-pQCT can be used for the in vivo assessment of Ct.Po. We confirm the use of BMD also for the local mapping of porosity on regions of cortical bone below $0.5 \mathrm{~mm}^{2}$ in size. In addition, we propose a rule for the cortical porosity estimation based on multiple parameters that are derived from HR-pQCT data. Applied to ex vivo samples, this method is more accurate than established BMD and threshold-based approaches.

\section{Conflicts of interest}

None.

\section{Acknowledgments}

This work was supported by the Deutsche Forschungsgemeinschaft (DFG Ra1380/9-1), by the Bundesministerium für Bildung und Forschung (BMBF, musculoskeletal research network 01EC1408L, subproject "characterization of cortical and subchondral bone"), by the German Academic Exchange Service (DAAD, grant no. 91578351), and by the Weijerhors Foundation (grant no. WH2). We acknowledge Mario Thiele for the data archiving, Dr. Daniel Rohrbach for the scripts for BMD histogram analysis and Dr. Ahmed BenSaïda for the Matlab implementation of the Shapiro-Wilk test.

\section{Appendix A. Automatic 3D registration of SAM and HR-pQCT images}

A custom algorithm for 3D registration of the HR-pQCT volume data to the SAM image was implemented in MATLAB ${ }^{\circ}$. First, 200 CT slices (100 above and 100 below, corresponding to a $6.09 \mathrm{~mm}$ high stack) were extracted around the approximate SAM cross-section. The slices were cropped around the femur shaft and rotated to match the orientation of the SAM image. A resized copy of the CT stack was generated, matching the in-plane SAM pixel size and the SAM image was padded with zeroes to match the size of the CT slice. The rigid transformation matrix aligning the central slice of the (resized) CT dataset to the SAM image was then calculated and applied to the extracted CT volume. This procedure yielded a CT stack with a center point aligned to the center point of the SAM image. After this, the SAM image and individual CT slices from the stack produced above were divided into 196 regions $(14 \times 14$, yielding a size of approx. $3 \mathrm{~mm}$ comparable with the average cortical thickness) for local 2D analysis of similarity; $x$ and $y$ coordinates of the midpoint of each region were stored for the next alignment steps. The $2 \mathrm{D}$ correlation coefficient, a widely used similarity measure for multimodality image registration [50,51], was calculated between each region of the SAM image and the corresponding region over the 200 CT slices; thus obtaining a $14 \times 14 \times 200$ map of similarity scores. For each region, a Gaussian model (a model with two peaks was used to exclude possible local outliers with strong similarity; model boundaries were set at the CT volume z-boundaries) was fit to the array of correlation coefficients corresponding to a single $x-y$ position and to 200 slices. Regions returning an invalid correlation coefficient (typically background regions) were excluded from the calculation. For each individual region, the Z-coordinates corresponding to the peak of the Gaussian fit were extracted, producing a 3D cloud of points located at positions of maximum similarity between the SAM image and the HR-pQCT slices. This 3D point cloud was then fitted with a plane model using the Ransac algorithm [52]. A distance threshold of $0.1 \mathrm{~mm}$ between the data points and the plane was used to discard fit outliers and the algorithm was stopped (determining the failure of the registration procedure) if a maximum number of 1000 trials was reached without converging to a useful solution. The 3D rigid transformation aligning the fitted plane with the $x-y$ plane was then computed and applied (using linear interpolation for the rotated voxels) to the HR-pQCT stack, producing a copy of the original HR-pQCT stack with its $x-y$ plane aligned with the SAM image plane. The final crosssectional image was then extracted from the rotated CT data at the new central z-coordinate of the fit plane. A last automatic 2D registration step was applied to the two-dimensional SAM and HR-pQCT images.

In order to quantify the potential impact of the inaccuracy of the registration on the correlation between local properties, we simulated registration errors by introducing fluctuations in the optimal transformation matrix, for each sample. Translational (magnitude $=\left[\begin{array}{llllll}0 & 1 & 2 & 4 & 8\end{array}\right.$ 12] [pixels]; random direction) and rotational (angle $=\left[\begin{array}{llll}0 & 0.1 & 0.2 & 0.3\end{array}\right.$ 0.4] [deg]) fluctuations were separately introduced and the local properties (BMD ${ }_{\text {mean }}$ and Ct.Po) pooled together for all samples. 1, 2, 4, 8 and 12 pixels translational fluctuations caused a relative increase of the $\mathrm{BMD}_{\text {mean }}$-Ct.Po RMSE of $+0.37 \%,+0.31 \%,+2.01 \%,+8.36 \%$ and $+14.76 \%$, respectively. Similarly, $0.1,0.2,0.3$ and 0.4 degrees of translational fluctuation led to a relative variation of the RMSE of $-0.14 \%,+0.95 \%,+1.64 \%$ and $+3.98 \%$, respectively.

\section{Appendix B. Supplementary data}

Supplementary data to this article can be found online at https:// doi.org/10.1016/j.bone.2018.05.028.

\section{References}

[1] Y. Carter, C.D.L. Thomas, J.G. Clement, A.G. Peele, K. Hannah, D.M.L. Cooper, Variation in osteocyte lacunar morphology and density in the human femur synchrotron radiation micro-CT study, Bone 52 (1) (2013) 126-132.

[2] N.J. Wachter, P. Augat, G.D. Krischak, M. Mentzel, L. Kinzl, L. Claes, Prediction of cortical bone porosity in vitro by microcomputed tomography, Calcif. Tissue Int. 68 (1) (2001) 38-42.

[3] J.D. Currey, R. Shahar, Cavities in the compact bone in tetrapods and fish and their effect on mechanical properties, J. Struct. Biol. 183 (2) (2013) 107-122.

[4] D.M.L. Cooper, C.E. Kawalilak, K. Harrison, B.D. Johnston, J.D. Johnston, Cortical bone porosity: what is it, why is it important, and how can we detect it? Curr. Osteoporos. Rep. 14 (5) (2016) 187-198.

[5] D.M.L. Cooper, C.D.L. Thomas, J.G. Clement, A.L. Turinsky, C.W. Sensen, B. Hallgrímsson, Age-dependent change in the 3D structure of cortical porosity at the human femoral midshaft, Bone 40 (4) (2007) 957-965.

[6] E. Seeman, Pathogenesis of bone fragility in women and men, Lancet 359 (9320) (2002) 1841-1850.

[7] Y. Bala, R. Zebaze, A. Ghasem-Zadeh, E.J. Atkinson, S. Iuliano, J.M. Peterson, S. Amin, A. Bjørnerem, L.J. Melton, H. Johansson, J.A. Kanis, S. Khosla, E. Seeman, Cortical porosity identifies women with osteopenia at increased risk for forearm fractures, J. Bone Miner. Res. 29 (6) (2014) 1356-1362.

[8] Y. Bala, Q.M. Bui, X.-F. Wang, S. Iuliano, Q. Wang, A. Ghasem-Zadeh, T.D. Rozental, M.L. Bouxsein, R.M.D. Zebaze, E. Seeman, Trabecular and cortical microstructure and fragility of the distal radius in women, J. Bone Miner. Res. 30 (4) (2015) 621-629.

[9] J.D. Currey, The effect of porosity and mineral content on the Young's modulus of elasticity of compact bone, J. Biomech. 21 (2) (1988) 131-139.

[10] M.B. Schaffler, D.B. Burr, Stiffness of compact bone: effects of porosity and density, J. Biomech. 21 (1) (1988) 13-16.

[11] M. Granke, Q. Grimal, A. Saïed, P. Nauleau, F. Peyrin, P. Laugier, Change in porosity is the major determinant of the variation of cortical bone elasticity at the millimeter scale in aged women, Bone 49 (5) (2011) 1020-1026.

[12] Y.N. Yeni, C.U. Brown, Z. Wang, T.L. Norman, The influence of bone morphology on fracture toughness of the human femur and tibia, Bone 21 (5) (1997) 453-459.

[13] J.D. Currey, Stress concentrations in bone, J. Cell Sci. s3-103 (61) (1962) 111-133.

[14] K.L. Bell, N. Loveridge, J. Power, N. Garrahan, B.F. Meggitt, J. Reeve, Regional differences in cortical porosity in the fractured femoral neck, Bone 24 (1) (1999) 57-64.

[15] G.R. Jordan, N. Loveridge, K.L. Bell, J. Power, N. Rushton, J. Reeve, Spatial clustering of remodeling osteons in the femoral neck cortex: a cause of weakness in hip fracture? Bone 26 (3) (2000) 305-313.

[16] A.J. Burghardt, H.R. Buie, A. Laib, S. Majumdar, S.K. Boyd, Reproducibility of direct quantitative measures of cortical bone microarchitecture of the distal radius and tibia by HR-pQCT, Bone 47 (3) (2010) 519-528.

[17] A. Ostertag, F. Peyrin, S. Fernandez, J.D. Laredo, M.C. de Vernejoul, C. Chappard, Cortical measurements of the tibia from high resolution peripheral quantitative computed tomography images: a comparison with synchrotron radiation microcomputed tomography, Bone 63 (2014) 7-14.

[18] A.J. Burghardt, G.J. Kazakia, S. Ramachandran, T.M. Link, S. Majumdar, Age- and gender-related differences in the geometric properties and biomechanical significance of intracortical porosity in the distal radius and tibia, J. Bone Miner. Res. 25 (5) (2010) 983-993.

[19] R. Zebaze, E. Seeman, Cortical bone: a challenging geography, J. Bone Miner. Res. 30 (1) (2015) 24-29. 
[20] R. Zebaze, A. Ghasem-Zadeh, A. Mbala, E. Seeman, A new method of segmentation of compact-appearing, transitional and trabecular compartments and quantification of cortical porosity from high resolution peripheral quantitative computed tomographic images, Bone 54 (1) (2013) 8-20.

[21] H. Follet, G. Boivin, C. Rumelhart, P.J. Meunier, The degree of mineralization is a determinant of bone strength: a study on human calcanei, Bone 34 (5) (2004) 783-789.

[22] C. Bergot, Y. Wu, E. Jolivet, L.Q. Zhou, J.D. Laredo, V. Bousson, The degree and distribution of cortical bone mineralization in the human femoral shaft change with age and sex in a microradiographic study, Bone 45 (3) (2009) 435-442.

[23] B.L. Jorgenson, H.R. Buie, D.D. Mcerlain, C. Sandino, S.K. Boyd, A comparison of methods for in vivo assessment of cortical porosity in the human appendicular skeleton, Bone 73 (2015) 167-175.

[24] E. Dall'Ara, B. Luisier, R. Schmidt, M. Pretterklieber, F. Kainberger, P. Zysset, D. Pahr, DXA predictions of human femoral mechanical properties depend on the load configuration, Med. Eng. Phys. 35 (11) (2013) 1564-1572.

[25] M.H. Fessy, B. Seutin, J. Béjui, Anatomical basis for the choice of the femoral implant in the total hip arthroplasty, Surg. Radiol. Anat. 19 (5) (1997) 283-286.

[26] E. Dall'Ara, P. Varga, D. Pahr, P. Zysset, A calibration methodology of QCT BMD for human vertebral body with registered micro-CT images, Med. Phys. 38 (5) (2011) 2602.

[27] K. Raum, K.V. Jenderka, A. Klemenz, J. Brandt, Multilayer analysis: quantitative scanning acoustic microscopy for tissue characterization at a microscopic scale, IEEE Trans. Ultrason. Ferroelectr. Freq. Control 50 (5) (2003) 507-516.

[28] S. Lakshmanan, A. Bodi, K. Raum, Assessment of anisotropic tissue elasticity of cortical bone from high-resolution, angular acoustic measurements, IEEE Trans. Ultrason. Ferroelectr. Freq. Control 54 (8) (2007) 1560-1570.

[29] K. Raum, J. Reißhauer, J. Brandt, Frequency and resolution dependence of the anisotropic impedance estimation in cortical bone using time-resolved scanning acoustic microscopy, J. Biomed. Mater. Res. 71A (3) (2004) 430-438.

[30] R. Hube, H. Mayr, W. Hein, K. Raum, Prediction of biomechanical stability after callus distraction by high resolution scanning acoustic microscopy, Ultrasound Med. Biol. 32 (12) (2006) 1913-1921.

[31] N. Otsu, A threshold selection method from gray-level histograms, IEEE Trans. Syst. Man Cybern. 9 (1) (1979) 62-66.

[32] L.M. Metcalf, E. Dall'Ara, M.A. Paggiosi, J.R. Rochester, N. Vilayphiou, G.J. Kemp, E.V. McCloskey, Validation of calcaneus trabecular microstructure measurements by HR-pQCT, Bone 106 (2018) 69-77.

[33] D.G. Altman, J.M. Bland, Measurement in medicine: the analysis of method comparison studies, J. R. Stat. Soc. Series D (The Statistician) 32 (1983) 307-317.

[34] E. Schileo, E. Dall'Ara, F. Taddei, A. Malandrino, T. Schotkamp, M. Baleani, M. Viceconti, An accurate estimation of bone density improves the accuracy of subject-specific finite element models, J. Biomech. 41 (11) (2008) 2483-2491.

[35] V. Bousson, C. Bergot, A. Meunier, F. Barbot, C. Parlier-Cuau, A.-M. Laval-Jeantet, J.-D. Laredo, CT of the middiaphyseal femur: cortical bone mineral density and relation to porosity, Radiology 217 (1) (2000) 179-187.

[36] G. Dougherty, A comparison of the texture of computed tomography and projection radiography images of vertebral trabecular bone using fractal signature and lacunarity, Med. Eng. Phys. 23 (2001) 313-321.

[37] L. Pothuaud, P. Carceller, D. Hans, Correlations between grey-level variations in 2D projection images (TBS) and 3D microarchitecture: applications in the study of human trabecular bone microarchitecture, Bone 42 (2008) 775-787.

[38] T. Lowitz, O. Museyko, V. Bousson, C. Chappard, L. Laouisset, J.-D. Laredo, K. Engelke, Advanced Knee Structure Analysis (AKSA): a comparison of bone mineral density and trabecular texture measurements using computed tomography and high-resolution peripheral quantitative computed tomography of human knee cadavers, Arthritis Res. Ther. 19 (1) (2017).

[39] A.J. Burghardt, J.-B. Pialat, G.J. Kazakia, S. Boutroy, K. Engelke, J.M. Patsch, A. Valentinitsch, D. Liu, E. Szabo, C.E. Bogado, M.B. Zanchetta, H.A. McKay, E. Shane, S.K. Boyd, M.L. Bouxsein, R. Chapurlat, S. Khosla, S. Majumdar, Multicenter precision of cortical and trabecular bone quality measures assessed by high-resolution peripheral quantitative computed tomography, J. Bone Miner. Res. 28 (3) (2013) 524-536.

[40] K. Raum, I. Leguerney, F. Chandelier, M. Talmant, A. Saïed, F. Peyrin, P. Laugier, Site-matched assessment of structural and tissue properties of cortical bone using scanning acoustic microscopy and synchrotron radiation $\mu \mathrm{CT}$, Phys. Med. Biol. 51 (3) (2006) 733-746.

[41] D.M.L. Cooper, C.D.L. Thomas, J.G. Clement, B. Hallgrímsson, Three-dimensional microcomputed tomography imaging of basic multicellular unit-related resorption spaces in human cortical bone, Anat. Rec. A: Discov. Mol. Cell. Evol. Biol. 288A (7) (2006) 806-816 \%U https://doi.org/10.1002/ar.a.20344/abstract.

[42] R.M.D. Zebaze, A. Ghasem-Zadeh, A. Bohte, S. Iuliano-Burns, M. Mirams, R.I. Price, E.J. Mackie, E. Seeman, Intracortical remodelling and porosity in the distal radius and post-mortem femurs of women: a cross-sectional study, Lancet 375 (9727) (2010) 1729-1736.

[43] F. Taddei, E. Schileo, B. Helgason, L. Cristofolini, M. Viceconti, The material mapping strategy influences the accuracy of CT-based finite element models of bones: an evaluation against experimental measurements, Med. Eng. Phys. 29 (9) (2007) 973-979.

[44] D. Garcia, P.K. Zysset, M. Charlebois, A. Curnier, A three-dimensional elastic plastic damage constitutive law for bone tissue, Biomech. Model. Mechanobiol. 8 (2) (2008) 149-165.

[45] M. Granke, A.J. Makowski, S. Uppuganti, M.D. Does, J.S. Nyman, Identifying novel clinical surrogates to assess human bone fracture toughness, J. Bone Miner. Res. 30 (7) (2015) 1290-1300.

[46] E. Bossy, M. Talmant, F. Peyrin, L. Akrout, P. Cloetens, P. Laugier, An in vitro study of the ultrasonic axial transmission technique at the radius: $1-\mathrm{MHz}$ velocity measurements are sensitive to both mineralization and intracortical porosity, J. Bone Miner. Res. 19 (9) (2004) 1548-1556.

[47] R.B. Ashman, S.C. Cowin, W.C. Van Buskirk, J.C. Rice, A continuous wave technique for the measurement of the elastic properties of cortical bone, J. Biomech. 17 (5) (1984) 349-361.

[48] T.V. Nguyen, J.R. Center, J.A. Eisman, Bone mineral density-independent association of quantitative ultrasound measurements and fracture risk in women, Osteoporos. Int. 15 (12) (2004) 942-947.

[49] W.P. Olszynski, J.D. Adachi, D.A. Hanley, K.S. Davison, J.P. Brown, Comparison of speed of sound measures assessed by multisite quantitative ultrasound to bone mineral density measures assessed by dual-energy X-ray absorptiometry in a large Canadian cohort: the Canadian multicentre osteoporosis study (CaMos), J. Clin. Densitom. 19 (2) (2016) 234-241.

[50] L. Dong, A.L. Boyer, An image correlation procedure for digitally reconstructed radiographs and electronic portal images, International Journal of Radiation Oncology, Biology and Physics 33 (5) (1995) 1053-1060.

[51] J. Kim, J.A. Fessler, Intensity-based image registration using robust correlation coefficients, IEEE Trans. Med. Imaging 23 (11) (2004) 1430-1444.

[52] P.D. Kovesi, MATLAB and Octave Functions for Computer Vision and Image Processing. 